\title{
A Behaviour and Awareness Disturbance: About Cerebral Lesions
}

\section{Formigo $\mathrm{M}^{*}$, Costa M, Martins J, Sarmento H and Cotter J}

Internal Medicine Department; Hospital Senhora da Oliveira, Portugal

*Corresponding author: Mariana Formigo Master Degree in Medicine, Internal Medicine Department, Hospital Senhora da Oliveira; Rua dos Cutileiros, 1144835-044 Creixomil, Guimaraes, Portugal, Tel: 00351253540330; Email: mariana.formigo@gmail.com

\section{Case Report}

Volume 4 Issue 2

Received Date: November 19, 2020

Published Date: December 07, 2020

DOI: $10.23880 /$ jidtm- 16000143

\section{Abstract}

A 67-year-old man presented with headache, anorexia and tonic-clonic seizures. He was previously hospitalized due to miliary tuberculosis and was currently medicated with 4-drug antituberculous therapy. At admission he was agitated, uncooperative, feverish, presenting no neurological deficits. His lumbar puncture revealed proteinorrhachia, normal white blood cells count and glucose levels. MRI revealed multiple small nodular contrast-enhanced lesions with vasogenic edema in both cerebellar hemispheres, protuberance, right thalamus, bilateral frontal and parietal cortex, and left temporalis and a cavitated with gadolinium ring enhancement. A month later, cerebral spinal fluid molecular test was positive for Mycobacterium tuberculosis (MT) complex and he is currently completing antibacillary treatment with good evolution.

Miliary tuberculosis may occur in both primary and reactivated tuberculosis, resulting from lymphohaematogenous dissemination of MT. Tuberculous meningitis and parenchymal lesions are the main presentations of CNS affection. The development of tuberculomas during appropriate antituberculous therapy is poorly understood and may correlate to an enhanced host immune response.

Keywords: Tuberculosis; Magnetic Resonance Imaging; Tuberculous Meningitis; Tuberculoma; Antituberculous Therapy

Abbreviations: TB: Tuberculosis; MT: Mycobacterium Tuberculosis; CSF: Cerebral Spinal Fluid; MRI: Magnetic Resonance Imaging; NAA: Nucleic Acid Amplification; HRZE: Isoniazid, Rifampin, Pyrazinamide and Ethambutol; HIV: Human Immune Virus; CT: Computed Tomography

\section{Introduction}

Disseminated tuberculosis (TB) usually occurs in immunocompromised subjects, infants and elderly and it have associated with significant morbidity and mortality [1]. It may occur as a progressive primary infection or as a reactivation of a latent focus through lymphohaematogenous dissemination of Mycobacterium tuberculosis (MT) [2,3].
A high clinical suspicion is required due to its frequent atypical presentation and diagnosis usually involves MT isolation in body fluids or biopsy specimens, nucleic acid amplification (NAA) molecular tests or cytohistopathological examination [1-3]. Considering extrapulmonary TB, specific specimens NAA testing should be performed due to its high specificity [4].

Central nervous system tuberculosis usually presents as tuberculous meningitis and parenchymal lesions (tuberculomas, abscesses and cerebritis) [5,6].

Neurologic clinical spectrum is broad and may vary from an asymptomatic or a vague prolonged ill health state to 
the development of meningeal irritation, focal brain lesions or increased intracranial pressure. The most frequent symptoms are non-specific including malaise, anorexia, fatigue, fever, myalgias, and headache and the most common clinical manifestations include cranial nerve deficits, altered mental states, hemiparesis, seizures and headache $[5,6]$.

Approximately $50 \%$ of children with tuberculous meningitis and $10 \%$ of adult patients present a prior history of tuberculosis [6].

\section{Case Presentation}

A 67-year-old man presented with headache, anorexia and tonic-clonic seizures. His medical background included a stable monoclonal gammopathy of undetermined significance and a previous hospitalization due to miliary tuberculosis, currently medicated with 4-drug antituberculous therapy with Isoniazid, Rifampin, Pyrazinamide and Ethambutol (HRZE).

At admission he was agitated, uncooperative, feverish, presenting no neurological deficits. His brain computed tomography was normal and a lumbar puncture revealed proteinorrhachia, normal white blood cells count and glucose levels. Acyclovir was empirically initiated.

Electroencephalography was normal; cerebral spinal fluid (CSF) fast molecular testing, microbiological cultures; Ziehl Neelsen and HIV screening test were negative.

Magnetic resonance imaging (MRI) revealed multiple small nodular contrast-enhanced lesions with vasogenic edema in both cerebellar hemispheres, protuberance, right thalamus, bilateral frontal and parietal cortex, and left temporalis (Figure 1) and a cavitated image (6 mm) with gadolinium ring enhancement surrounded by edema and erasure of the left medial parieto-occipital region grooves (Figures $2 \& 3$ ).

He initiated corticosteroids with dexamethasone and maintained four-drug antibacillary therapy with HRZE for 2 months, being later discharged with complete regression of his neurological symptoms, forwarded to Internal Medicine consultation.

A month later, CSF molecular test was positive for Mycobacterium tuberculosis complex and he is currently completing two-drug treatment with Isoniazid, Rifampin with good clinical evolution.

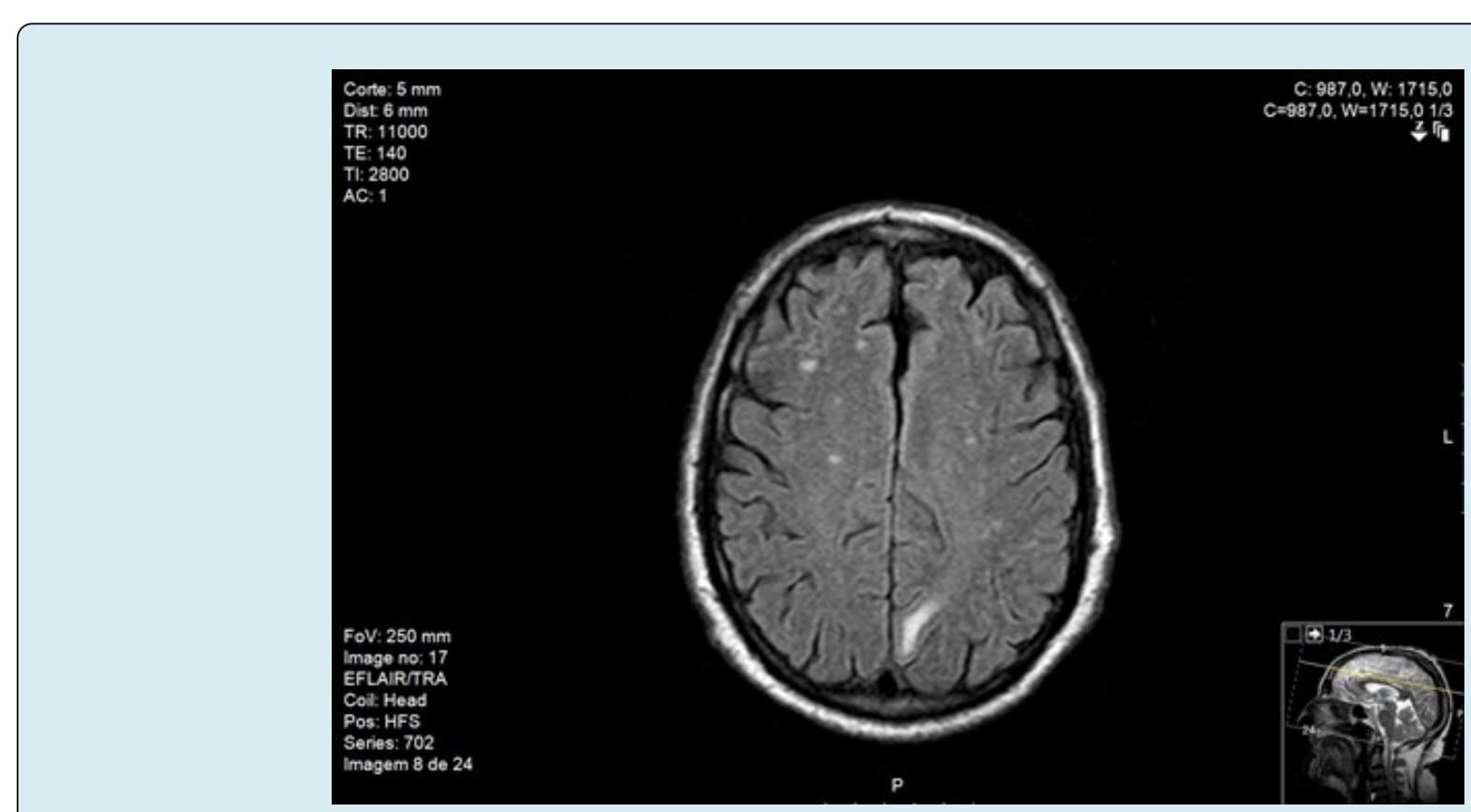

Figure 1: MRI revealing multiple small nodular contrast-enhanced lesions with vasogenic edema in both frontal and parietal cortex, and left temporalis. 


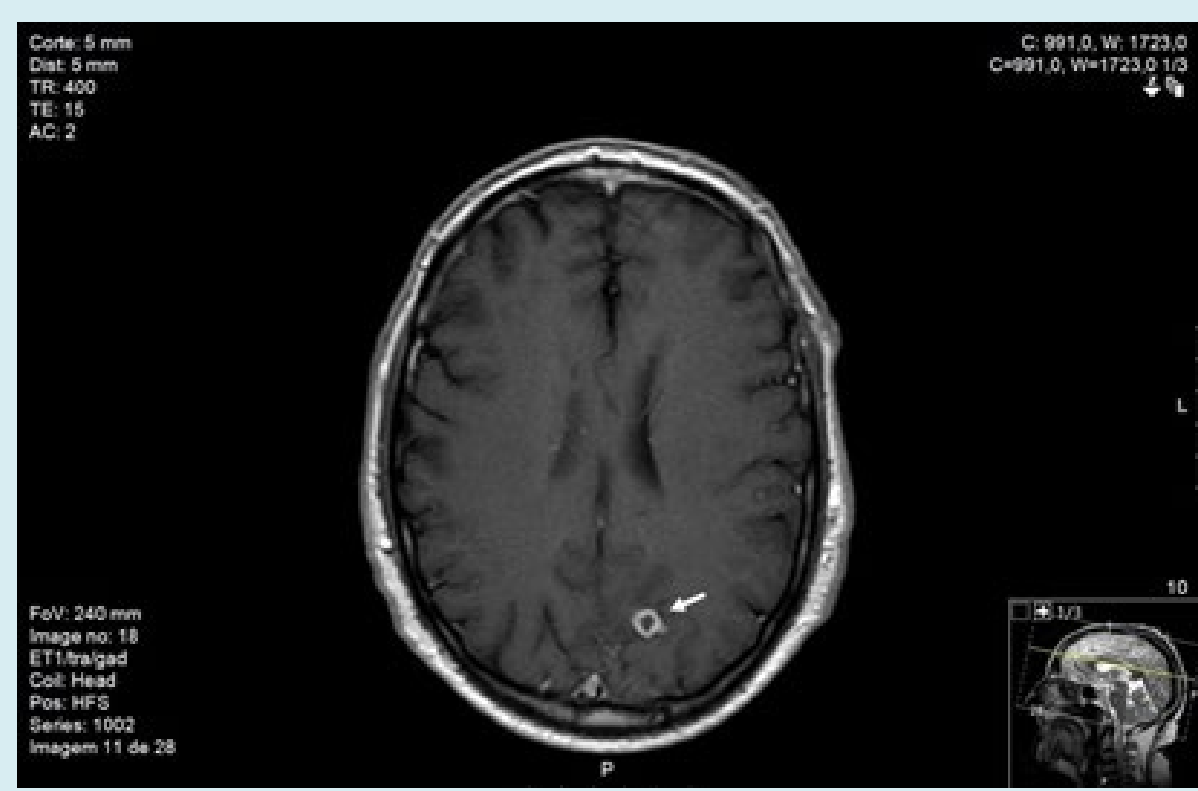

Figure 2: MRI revealing a cavitated image (arrow) with gadolinium ring enhancement surrounded by edema and erasure of the left medial parieto-occipital region grooves.

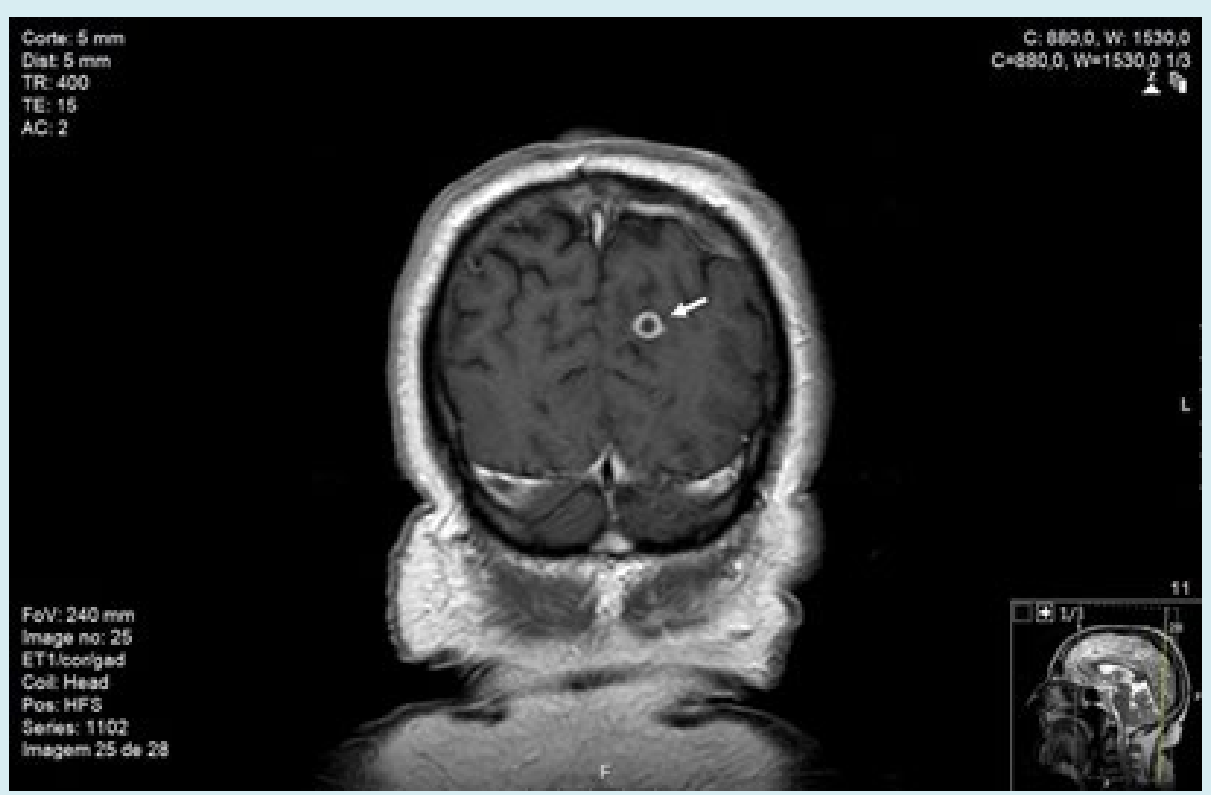

Figure 3: MRI revealing a cavitated image (arrow) with gadolinium ring enhancement surrounded by edema and erasure of the left medial parieto-occipital region grooves.

\section{Discussion}

The development of tuberculomas during appropriate antibacillary therapy is poorly understood and may be associated with an enhanced host immune response [5].

Imaging plays an important role in the diagnosis of central nervous system, especially MRI by providing better quality images than Computed Tomography (CT) [6]. Meningeal tuberculosis usually reveals thickening and intense enhancement of meninges, especially in basilar regions, ventricular enlargement, and infarcts. Multiple intracranial tuberculomas are frequent when tuberculous meningitis is part of miliary tuberculosis. Tuberculomas are firm, avascular, spherical granulomatous masses, may vary in number and size and usually distribute to frontal 
and parietal zones. Surrounding brain tissue is usually compressed around the lesion and shows oedema and gliosis. Tuberculomas may contain necrotic areas composed of caseous material, in which MT can be demonstrated. [5,6].

Prompt TB diagnosis and antibacillary treatment is crucial owing to its associated significant morbidity and mortality $[2,7]$.

Chemotherapy for tuberculous meningitis is initiated with a four-drug therapy with isoniazid, rifampin, pyrazinamide and ethambutol for 2 months followed by a two-drug antibacillary therapy with isoniazid and rifampin for the remaining 7-10 months. Adjunctive corticosteroid therapy with dexamethasone or prednisolone tapered over 6-8 weeks is recommended as it seems to be associated with less mortality $[4,7,8]$.

Brain tuberculomas may completely disappear after therapy, although residual calcifications may persist in up to a quarter of patients [6].

TB has been well known for centuries, although it is still prevalent in developing and well-developed countries nowadays and it can present unusual presentations. Its natural course can lead to great morbidity and death, so it is important to establish prompt diagnosis and treatment to improve its prognosis.

\section{References}

1. Kim JH, Langston AA, Gallis HA (1990) Miliary tuberculosis: epidemiology, clinical manifestations, diagnosis, and outcome. Rev Infect Dis 12(4): 583-590.
2. Sharma SK, Mohan A (2017) Miliary tuberculosis. Microbiol Spectr 5(2).

3. Sharma SK, Mohan A, Sharma A, Mitra DK (2005) Miliary tuberculosis: new insights into an old disease. Lancet Infect Dis 5(7): 415-430.

4. Lewinsohn DM, Leonard MK, LoBue PA, David L Cohn, Charles L Daley, et al. (2017) Official American Thoracic Society/Infectious Diseases Society of America/Centers for Disease Control and Prevention Clinical Practice Guidelines: Diagnosis of Tuberculosis in Adults and Children. Clin Infect Dis 64(2): 111-115.

5. Nicolls DJ, King M, Holland D, Bala J, del Rio C (2005) Intracranial tuberculomas developing while on therapy for pulmonary tuberculosis. Lancet Infect Dis 5(12): 795-801.

6. Garg RK (1999) Tuberculosis of the central nervous system. Postgrad Med J 75(881): 133-140.

7. Nahid P, Dorman SE, Alipanah N, Pennan M Barry, Jan L Brozek, et al. (2016) Official American Thoracic Society/ Centers for Disease Control and Prevention/Infectious Diseases Society of America Clinical Practice Guidelines: Treatment of Drug-Susceptible Tuberculosis. Clin Infect Dis 63(7): e147-195.

8. Antunes A, Carvalho A, Gonçalves A, Betaina Ferreira, Carla Ribeiro, et al. (2013) Manual de Tuberculose e micobactérias não tubeculosas. Duarte R, Brito MC, Villar M, Correia AM (Eds.), Programa nacional para a tuberculose. DGS, Portugal. 